\title{
Engineering strategy of yeast metabolism for higher alcohol production
}

\author{
Fumio Matsuda ${ }^{1}$, Chikara Furusawa ${ }^{2}$, Takashi Kondo ${ }^{1}$, Jun Ishii ${ }^{1}$, Hiroshi Shimizu ${ }^{2}$ and Akihiko Kondo ${ }^{3^{*}}$
}

\begin{abstract}
Background: While Saccharomyces cerevisiae is a promising host for cost-effective biorefinary processes due to its tolerance to various stresses during fermentation, the metabolically engineered S. cerevisiae strains exhibited rather limited production of higher alcohols than that of Escherichia coli. Since the structure of the central metabolism of $S$. cerevisiae is distinct from that of $E$. coli, there might be a problem in the structure of the central metabolism of $S$. cerevisiae. In this study, the potential production of higher alcohols by S. cerevisiae is compared to that of E. coli by employing metabolic simulation techniques. Based on the simulation results, novel metabolic engineering strategies for improving higher alcohol production by S. cerevisiae were investigated by in silico modifications of the metabolic models of $S$. cerevisiae.

Results: The metabolic simulations confirmed that the high production of butanols and propanols by the metabolically engineered E. coli strains is derived from the flexible behavior of their central metabolism. Reducing this flexibility by gene deletion is an effective strategy to restrict the metabolic states for producing target alcohols. In contrast, the lower yield using S. cerevisiae originates from the structurally limited flexibility of its central metabolism in which gene deletions severely reduced cell growth.

Conclusions: The metabolic simulation demonstrated that the poor productivity of $S$. cerevisiae was improved by the introduction of E. coli genes to compensate the structural difference. This suggested that gene supplementation is a promising strategy for the metabolic engineering of S. cerevisiae to produce higher alcohols which should be the next challenge for the synthetic bioengineering of $S$. cerevisiae for the efficient production of higher alcohols.
\end{abstract}

\section{Background}

The bioproduction of higher alcohols with more than 3 carbon atoms is desirable because they have the preferred properties of renewable liquid fuels and wide applications in commodity chemicals. In designing fermentation processes for mass production, the development of recombinant microbial strains is a critical first step [1]. The pioneering achievements were demonstrated by the construction of metabolically engineered Escherichia coli strains that produce butanols and propanols. Due to insufficient genetic tools for the natural 1-butanol producer clostridia [2], E. coli has been engineered for 1-butanol fermentation by introducing the CoA-dependent clostridial pathway [3-7]. The production of 1-butanol

\footnotetext{
* Correspondence: akondo@kobe-u.ac.jp

${ }^{3}$ Department of Chemical Science and Engineering, Graduate School of Engineering, Kobe University, Rokkodaicho, Nada-ku, Kobe, Hyogo, Japan Full list of author information is available at the end of the article
}

was preliminary achieved in the range of 0.55 to $1.2 \mathrm{~g} / \mathrm{L}$ $[4,6,8]$. The maximum 1-butanol titer $(30 \mathrm{~g} / \mathrm{L})$ was achieved by the introduction of irreversible transenoylCoA reductase and the creation of NADH and acetylCoA driving forces $[9,10]$. The production of higher alcohols, including 1-propanol, 1-butanol, isobutanol, 2methyl-1-butanol, and 3-methyl-1-butanol, was also demonstrated by introducing the keto-acid pathway [8,11-22]. This implementation resulted in 1-butanol production of approximately $0.85 \mathrm{~g} / \mathrm{L}$ [11]. In these studies, the production of the target alcohols was achieved by the introduction of the foreign genes required for their biosyntheses and by gene deletions to modulate the entire metabolism of the strains.

Based on these successes, a metabolic engineering study was initiated in baker's yeast (Saccharomyces cerevisiae). Yeast is a promising host for cost-effective biorefinary processes due to its tolerance to various stresses 
during fermentation. Tolerance to high concentrations of alcohol should be useful for the industrial production of higher alcohols [23-28]. The constructed yeast strains, however, exhibited limited production of higher alcohols. For instance, engineered S. cerevisiae expressing enzymes involved in the CoA-depending clostridial pathway yielded only $2.5 \mathrm{mg} / \mathrm{L}$ of 1 -butanol [29]. One technical reason for the low yield is the reduced activity of the introduced enzymes due to the ectopic expression of the bacterial genes. Another possible reason is that there might be a problem in the structure of the central metabolism of $S$. cerevisiae. The structure of the central metabolism of $S$. cerevisiae is distinct from that of $E$. coli in terms of the anaplerotic pathways, cytosolic acetyl-CoA (AcCoA) biosynthesis, fermentation products, and occurrence of mitochondria (Figure 1). For the mass production of higher alcohols, these metabolic pathways must be modified to increase the metabolic flow towards the synthesis of the target alcohols. This means that the precursor synthesis, cofactor regeneration, and supply of the building blocks for active cell growth must be simultaneously achieved by the central metabolisms while maintaining the carbon and redox

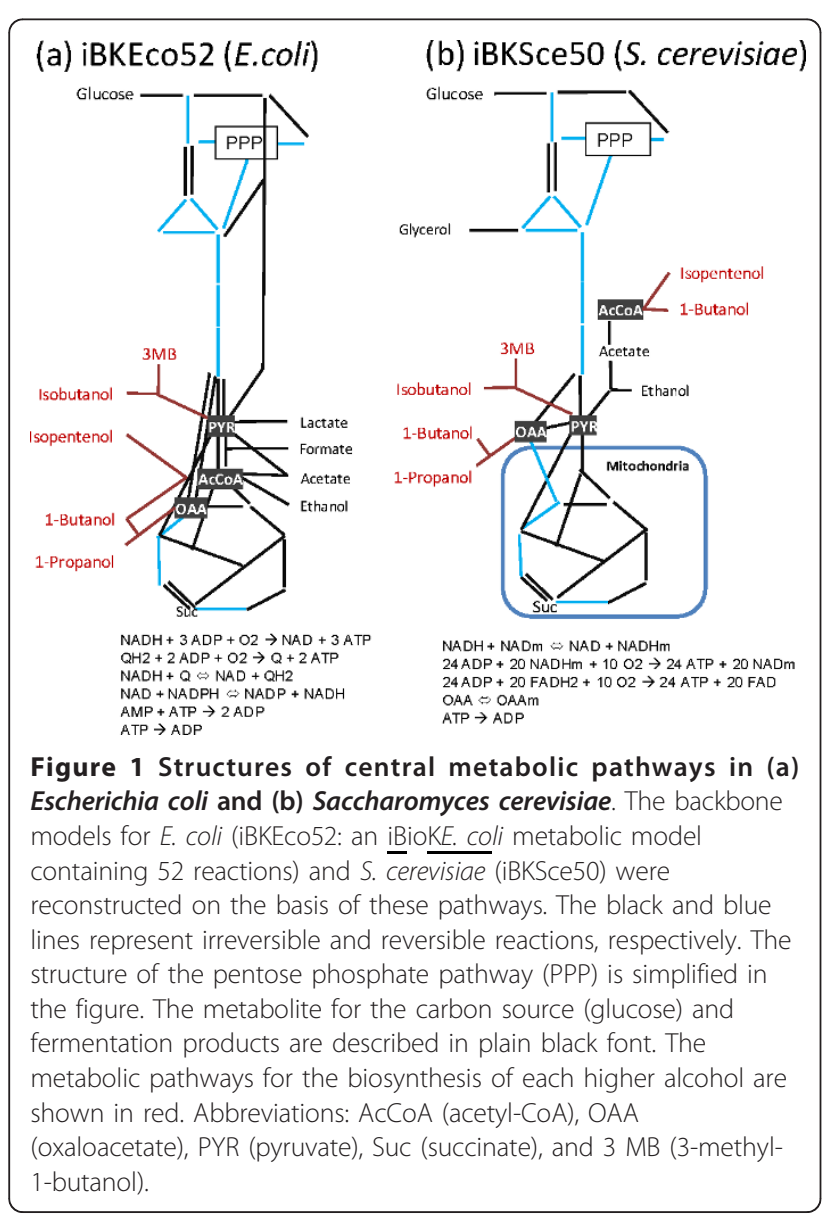

balances. Because the range of possible distributions of metabolic fluxes is restricted by the structure of the central metabolic network, S. cerevisiae may not be able to supply the required amount of the precursor and the reducing powers needed for the biosynthesis of higher alcohols. In such cases, the engineering strategy has to be revised by further modification of $S$. cerevisiae metabolism.

In this study, the potential production of higher alcohols by $S$. cerevisiae is compared to that of E. coli by employing in silico metabolic simulation techniques based on flux balance analysis (FBA). FBA is a mathematical approach for analyzing the flow of metabolites through a metabolic network [30-32]. The distribution of metabolic fluxes in the metabolic networks can be analyzed using the stoichiometric model of the metabolic reactions without knowledge of the metabolite concentrations or details of the enzyme kinetics [33,34]. Although FBA assumes the ideal metabolism conditions by ignoring all dynamic aspects of metabolic regulations such as gene expression, feedback regulation, and posttranslational modifications, it has been demonstrated that FBA can evaluate the performance of a metabolic network [30,33]. In this report, FBA-based metabolic simulation was demonstrated by following the strategy applied for the actual metabolic engineering. After reactions required for the biosynthesis of higher alcohols were introduced into the metabolic models, FBA-based metabolic simulations were performed for multiple gene-deletion mutants to evaluate the capabilities of the engineered strains to produce higher alcohols. For this purpose, the backbone metabolic models, which describe the essential structures of their central metabolism (Figure 1), were employed to reduce the number of genedeletion combinations to be tested. By using the small backbone models, the performance of all single, double, triple, and quadruple deletion mutants could be comprehensively investigated within a realistic period. The in silico analysis was applied for the backbone models of both $E$. coli and S. cerevisiae to highlight differences between their metabolic networks. The comparative analyses revealed that the central metabolism of S. cerevisiae has limited potential for the efficient production of higher alcohols in comparison with that of E. coli. It is also predicted that its poor productivity can be improved by extension of the central metabolic network.

\section{Results}

\section{Construction of backbone models}

The backbone metabolic models of E. coli and S. cerevisiae were employed for this analysis. The backbone metabolic models, which describe the essential structures of the central metabolism, were constructed from information from both the literature [35-41] and 
databases [42-44] (Figure 1 and Additional file 1). The backbone models for E. coli and S. cerevisiae are hereafter designated as iBKEco52 (i.e., an iBioKE. coli metabolic model containing 52 reactions) and iBKSce50, respectively. In iBKSce50, the metabolic network is compartmentalized in the cytosol and mitochondria. Although the backbone models contain only 50 or 52 reactions, their behavior was similar to that of genomescale models of E. coli (iJR904) and S. cerevisiae (iMM904)[45,46] (Additional file 2). These original models were modified by the addition of reactions for the synthesis of each higher alcohol. As shown in Figure 1 , the following 6 pathways were tested: (1) 1-propanol by oxaloacetate (OAA), (2) 1-butanol by AcCoA, (3) 1butanol by OAA, (4) isobutanol by pyruvate (PYR), (5) 3-methylbutanol by PYR, and (6) isopentenol from AcCoA (Additional file 3). By using these models, the potential production capabilities of higher alcohols were compared between E. coli and S. cerevisiae.

\section{Metabolic simulation of reaction-deleted mutants of $E$. coli}

As the first step of the analysis, the metabolic performance of E. coli was evaluated as a reference for the comparison. The potential productions of higher alcohols by $E$. coli were determined by employing the FBAbased metabolic simulations. In the procedure, the metabolic flux distribution was optimized to maximize the cell growth rate [30]. It is based on the assumption that a microbial metabolic system should evolve towards faster cell growth [47]. As this assumption has been experimentally supported [48], it is expected that the product yield will be improved when the production of higher alcohols contributes to faster cell growth. Here, the FBA-based metabolic simulations were performed by maximizing metabolic flux for biomass production from glucose using the linear optimization method. The results revealed that no higher alcohols were produced from glucose in the all modified backbone models of $E$. coli. The low productivity demonstrated by the in silico metabolic models mirrors the poor performance of actual recombinant $E$. coli strains just after the introduction of genes required for higher alcohol biosynthesis $[4,8,29]$.

To improve the yield of the target alcohols, the deletion of metabolism-related genes has been demonstrated to regulate the flux balances in the metabolic network $[8,11]$. The genes, however, must be deleted with regard to both the product yield and cell growth because gene deletion often seriously hampers cell growth. Because the effects of gene deletions could not be predicted empirically, FBA-based metabolic simulations were performed to elucidate the optimal combination of genes to be deleted [49-52].
Via this methodology, the production of higher alcohols by E. coli was investigated by performing FBAbased metabolic simulations for all single, double, triple, and quadruple deletion mutants generated from the metabolic model (see Methods for the detailed procedure). In the analysis, reactions instead of genes were removed from the metabolic models. The simulation results were verified by comparisons with literature data. The metabolic engineering of $E$. coli demonstrated that the product yield of a recombinant $E$. coli strain expressing genes for 1-butanol or isobutanol biosynthesis was enhanced by deletions of genes such as $\triangle \mathrm{adhE}, \triangle \mathrm{ldhA}$, $\triangle f r d B C$, and $\triangle$ pta as well as $\triangle \mathrm{adhE}, \triangle l d h A, \triangle f r d B C$, and $\triangle \mathrm{pfl}$, respectively $[4,8]$. The results were reproduced by FBA-based metabolic simulations using the modified backbone models. The 1-butanol and isobutanol yields were increased to 0.14 and $0.19\left(\mathrm{Y}_{\mathrm{Cmol} / \mathrm{Cmol} \text { glucose }}\right)$, respectively, by the deletions of the corresponding reactions.

In the case of the metabolic simulation for 1-butanol production, the growth rates and product yields were predicted for 213,052 multiple deletion models generated from the E. coli backbone model. Among the 8,668 viable and 1-butanol-producing models, an elite 347 models were considered "proper strains" in which all deletions contributed to an improvement in the 1-butanol yield (Table 1 and Additional file 4). The performances of the proper strains were compared with respect to the growth speed ( $\mathrm{x}$-axis) and 1-butanol yield (y-axis), as shown in Figure 2a. Although a negative correlation between the growth rate and 1-butanol yield was observed, several actively growing strains produce a relatively large amount of 1-butanol. Because these preferable strains are candidate targets for metabolic engineering, these results confirmed that the central metabolism of E. coli has promising potential for 1-butanol production as experimentally demonstrated $[9,10]$. Similar trends were observed for isobutanol (Figure 2c), 1-propanol (Figure 2e), and all other pathways (Additional file 5).

Using the same dataset, the impact of each deletion was evaluated. The improvement in 1-butanol yield and the loss of cell growth caused by each deletion were determined for all proper strains. The effects were averaged for each reaction, and the results are shown in Figures 3a-c. The $x$ - and $y$-axes represent the average loss of cell growth and average gain of product yield, respectively, caused by deleting the reaction. The results indicated that deletion of genes related to anaerobic fermentation, such as $l d h A$ and $a d h E$, increased the product yield without decreasing cell growth. Other genes, such as $p g i$, tended to increase the product yield but reduce cell growth. These results rationalize the strategy employed for the metabolic engineering of E. coli. After 
Table 1 The numbers of proper strains among all single, double, triple, and quadruple deletion mutants generated from the backbone models for E. coli (iBKEco52) and S. cerevisiae (iBKSce50, iBKSce50 $\Delta$ mit, and iBKSce50+7)

\begin{tabular}{lllll}
\hline Target higher alcohols & \multicolumn{4}{l}{$\begin{array}{l}\text { Number of proper strains } \\
\text { Total (single/double/triple/quadruple deletion mutants) }\end{array}$} \\
\cline { 2 - 5 } & iBKEco52 & iBKSce50 & iBKSce50 $\mathbf{m i t}$ & iBKSce50+7 \\
\hline 1-propanol from OAA & 458 & 40 & 108 & 467 \\
& $(0 / 11 / 85 / 362)$ & $(2 / 16 / 14 / 8)$ & $(4 / 27 / 50 / 27)$ & 192 \\
1-Butanol from AcCoA & 347 & 1 & 49 & $(33 / 22 / 44 / 93)$ \\
& $(1 / 17 / 87 / 242)$ & $(1 / 0 / 0 / 0)$ & $(2 / 7 / 17 / 23)$ & 373 \\
1-Butanol from OAA & 501 & 71 & 120 & $(2 / 17 / 116 / 238)$ \\
Isobutanol from PYR & $(0 / 10 / 89 / 402)$ & $(0 / 10 / 30 / 31)$ & $(3 / 25 / 59 / 33)$ & 276 \\
& 501 & 38 & 85 & $(2 / 18 / 81 / 175)$ \\
3-Methyl-1-butanol from PYR & $(1 / 14 / 83 / 403)$ & $(2 / 15 / 21 / 0)$ & $(4 / 25 / 30 / 26)$ & 94 \\
& 208 & 46 & 98 & $(2 / 5 / 33 / 54)$ \\
Isopentenol from AcCoA & $(2 / 13 / 51 / 142)$ & $(1 / 15 / 21 / 9)$ & $(4 / 25 / 46 / 23)$ & 166 \\
& 330 & 0 & 104 & $(2 / 10 / 54 / 100)$ \\
\hline
\end{tabular}

The "proper" strains were defined as viable target-producing strains in which all deletions contributed to the improvement of product yields. The growth rates and product yields were determined by FBA-based metabolic simulations. iBKSce $50 \Delta$ mit is an hypothetical S. cerevisiae model derived by merging the cytosolic and mitochondrial networks into one compartment. iBKSce $50+7$ is a model of iBKSce50 expanded by the addition of 7 E. coli reactions.

the construction of a metabolic pathway for higher alcohol biosynthesis, the product yields were improved by deleting genes while accounting for the impact on cell growth.

\section{Metabolic simulation of reaction-deleted mutants of S. cerevisiae}

In order to evaluate a performance of the in silico analysis using the backbone model of $S$. cerevisiae, iBKSce50, predicted growth rates of all single-reaction-deleted mutants were compared with the experimental data. The published phenotype data demonstrated that an inviable phenotype was observed for single gene-deleted mutants including $\Delta p g i 1, \Delta f b a 1, \Delta t p i 1, \Delta p g k 1, \Delta p y k 1$, and $\Delta r k i 1$ among the genes included in iBKSce 50 [53-55]. Most results could be reproduced by the FBAbased metabolic simulation using iBKSce50 (Additional file 6). By using the backbone model of $S$. cerevisiae, iBKSce50, the potential metabolic performance of $S$. cerevisiae was also evaluated using the same procedure for $E$. coli, and the results were compared with that of $E$. coli. The FBA-based metabolic simulations for all single, double, triple, and quadruple deletion mutants indicated that the number of proper strains was smaller than that for $E$. coli for all target alcohols (Table 1). In particular, for the case of acetyl-CoA-derived higher alcohols, no or very few target-producing strains were observed (Figure $2 \mathrm{a}$ and Table 1). Exceptionally good results were observed for isobutanol, probably because the redox balance required for isobutanol biosynthesis is similar to that for ethanol (Figure 2c). In the case of the other pathways, such as that towards 1-propanol, actively growing and target-producing strains were not observed among the proper strains (Figure 2e). It was also demonstrated few genes could be deleted without decreasing cell growth (Figure 3d-e). These results suggest that the strategy employed for the engineering of $E$. coli metabolism should not be effective for a construction of $S$. cerevisiae over-producing higher alcohols.

\section{Properties of the metabolic network}

The in silico simulations suggest that the central metabolism of $E$. coli could take many states of flux distribution because there are large variations in yield and growth performance among deletion strains (Figures 2ac). In contrast, the central metabolism of $S$. cerevisiae has rather limited or restricted metabolic behaviors. To support these observations, the natures of $E$. coli and $S$. cerevisiae metabolic networks were compared using elementary mode analysis. The analysis decomposes a complex metabolic network into many pathway subsets comprising a minimal set of enzymes that can support the steady-state operation of cellular metabolism (elementary mode)[33,36,56]. Because 1 elementary mode represents an independent cellular physiological state, the number of generated elementary modes reflects the flexibility of the metabolic network. The numbers of elementary modes of the E. coli and S. cerevisiae backbone models were 34,880 and 690 respectively, suggesting that the behavior of the central metabolism of $S$. cerevisiae is more restricted than that of E. coli (Table 2).

As the difference should be derived from the nature of the metabolic networks, 2 structural properties-network density and characteristic path length-were compared between the E. coli and $S$. cerevisiae backbone models $[56,57]$. Here, a substrate-product pair was considered a pair of nodes connected by an undirected edge. The network density represents the density of reactions among 

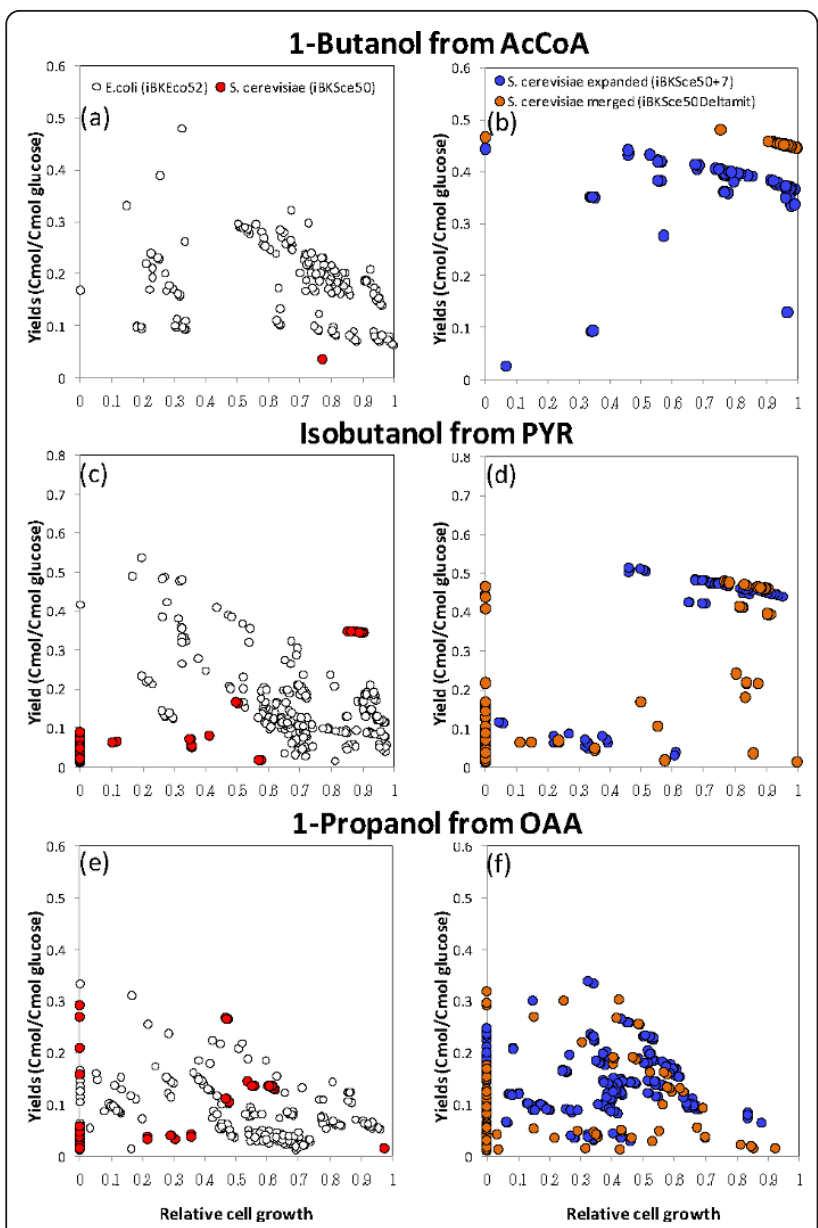

Figure 2 Metabolic simulations of multiple deletion mutants generated from the backbone models of $E$. coli (iBKEco52) and S. cerevisiae (iBKSce50, iBKSce50 $\Delta \mathrm{mit}$, and iBKSce50+7). The product yield and cell growth performance of the proper strains are shown for the production of 1-butanol from AcCoA ( $a$ and b), isobutanol from PYR (c and d), and 1-propanol from OAA (e and f). Open circles represent the data obtained from E. coli (iBKECo50); closed red, blue, and orange circles represent the data of original (iBKSce50), merged (iBKSce50 $\triangle$ mit), and expanded (iBKSce50+7) models of $S$. cerevisiae, respectively. iBKSce $50 \Delta$ mit is an hypothetical S. cerevisiae model derived by merging the cytosolic and mitochondrial networks into one compartment. iBKSce50+7 is a model of iBKSce50 expanded by the addition of 7 E. coli reactions.

The cell growth rate data were represented by relative values. A cell growth rate level determined from the wild type model was arbitrary set at 1 .

metabolites, and it should be 1 when all metabolites are connected with each other. The observed network densities of the E. coli and S. cerevisiae backbone models were 0.117 and 0.092 , respectively, indicating that the metabolites in the E. coli network are more densely connected by the metabolic reactions (Table 2). The characteristic path length indicates the average distances of all of the metabolite-metabolite pairs. For example, the distance between glucose and glucose-6-phosphate is 1 because they are directly connected by the reaction catalyzed by hexokinase. The determined characteristic path lengths suggest that the metabolites in E. coli are more closely connected, as the characteristic path length of $E$. coli is smaller than that of S. cerevisiae (Table 2). Although a relationship between those properties and higher alcohol production is unclear, the results suggest that the smaller number of elementary modes of $S$. cerevisiae backbone model is, at least in part, derived from the lesser network density and longer characteristic path length of the metabolic network.

\section{Improvement of the higher alcohol productivity of S. cerevisiae by the addition of $E$. coli genes}

To increase the network density and reduce the characteristic path length of the central metabolic network of $S$. cerevisiae, the metabolic structure was modified by considering 2 structural features. Because the metabolic networks of S. cerevisiae are compartmentalized into the cytosol and mitochondria, translocations of $\mathrm{NADP}^{+}$, $\mathrm{NADPH}$, and acetyl-CoA between these compartments were prohibited in the backbone model (Figure 1). Here, a hypothetical metabolic network of $S$. cerevisiae was constructed by merging the cytosolic and mitochondrial networks and was designated iBKSce $50 \Delta$ mit. The network density and characteristic path length of iBKSce $50 \Delta$ mit were improved to 0.113 and 2.664, respectively, by which the number of elementary modes was also increased to 5,859 (Table 2). Another structural difference between $E$. coli and S. cerevisiae is the occurrence of various shortcuts in $E$. coli such as pyridine nucleotide transhydrogenase, the anaplerotic pathways, the Entner-Doudoroff pathway, and acetyl-CoA synthesis by pyruvate formate-lyase. To supplement these metabolic functions in $S$. cerevisiae, the $7 \mathrm{E}$. coli reactions encoded by $p n t A B$, edd, pfl, pps, maeD, ppc, and $m d h$ were added to the backbone metabolic model of $S$. cerevisiae as cytosolic reactions. The expanded model was designated as iBKSce50+7. Other reactions, such as those related to the TCA cycle and those catalyzed by large protein complexes, were arbitrarily removed from the inserted reactions. The expanded model exhibited improved network properties and a larger number of elementary modes (Table 2).

To test the effects of those modifications, the FBAbased metabolic simulations were performed again using iBKSce $50 \Delta$ mit and iBKSce50+7 (Figure 2b, $d$ and 2f). The results revealed that the metabolic performances of these models were improved in terms of the numbers of proper strains (Table 1 ) as well as the target production (Figure $2 \mathrm{~b}, \mathrm{~d}$ and $2 \mathrm{f}$ ). For instance, the deletion strains of iBKSce $50 \Delta$ mit $\Delta p d c\left(\mathrm{Y}_{\mathrm{Cmol} / \mathrm{Cmol} \text { glucose: }} 0.445\right.$, relative growth rate: 1.0) and iBKSce50+7 $\Delta p g i\left(\mathrm{Y}_{\mathrm{Cmol} / \mathrm{Cmol} \text { glucose: }}\right.$ 0.347 , relative growth rate: 0.88 ) were able to produce 

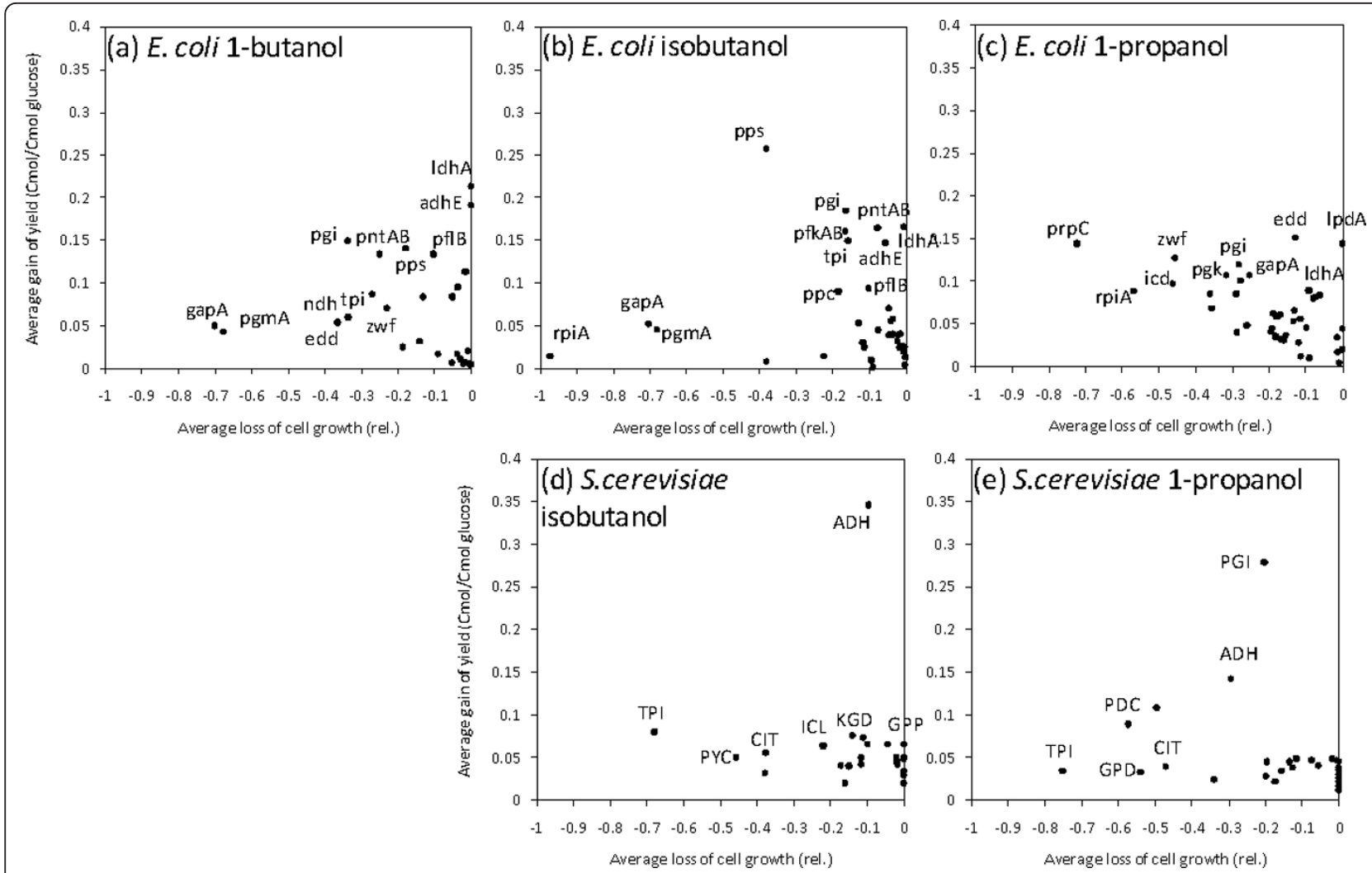

Figure 3 Effects of the deletion of each reaction in the modified backbone models of E. coli (iBKEco52) and S. cerevisiae (iBKSce50)) Average loss of cell growth and average gain of product yields caused by the deletion of each reaction in the modified backbone models of $E$. coli for the production of (a) 1-butanol by AcCOA, (b) isobutanol by PYR, and (c) 1-propanol by OAA are shown in the figure. Effects of the modified backbone models of S. cerevisiae for producing (d) isobutanol and (e) 1-propanol are also presented. The results for the production of 1-butanol by AcCoA by S. cerevisiae are not shown because there was only 1 target-producing model.

1-butanol with better yields than the most of $E$. coli models (Figure 2d). The comparison of the flux distributions at the fixed oxygen consumption rate condition revealed that although the metabolic balance required for 1-butanol biosynthesis could not been attained by the original S. cerevisiae model (iBKSce50, Figure 4a), a significant amount of 1-butanol could be generated in the merged model iBKSce50 $\Delta$ mit $\Delta p d c$ (Figure $4 \mathrm{~b}$ ) and in the expanded model (iBKSce50+7 $\Delta p g i$, Figure $4 \mathrm{c}$ ) by

Table 2 The network properties of the backbone models for E. coli (iBKEco52) and S. cerevisiae (iBKSce50, iBKSce50 $\Delta$ mit, and iBKSce50+7)

\begin{tabular}{|c|c|c|c|c|}
\hline \multirow[t]{2}{*}{ Network properties } & \multicolumn{4}{|c|}{ Backbone models } \\
\hline & iBKEco52 & iBKSce50 & iBKSce $50 \Delta$ mit & $\begin{array}{l}\text { iBKSce } 50 \\
+7 \\
\end{array}$ \\
\hline Number of nodes & 44 & 50 & 44 & 52 \\
\hline Network density & 0.117 & 0.092 & 0.113 & 0.095 \\
\hline $\begin{array}{l}\text { Characteristic path } \\
\text { length }\end{array}$ & 2.577 & 2.92 & 2.664 & 2.790 \\
\hline $\begin{array}{l}\text { Number of } \\
\text { elementary modes }\end{array}$ & 34,880 & 690 & 5,859 & 25,427 \\
\hline
\end{tabular}

using the modified metabolic functions in these models. Similar preferable results were observed for all pathways investigated (Figure 2d and $2 \mathrm{f}$ and Supplementary Data S5).

\section{Discussion}

The comparative in silico analyses performed in this study indicated that E. coli and S. cerevisiae have distinct potentials for higher alcohol production that originate from structural differences in their central metabolic networks (Figure 1). As demonstrated by the FBA-based metabolic analyses, the behavior of the central metabolism of E. coli appears more flexible than that of S. cerevisiae. Here, the flexibility of the metabolic network indicates the variety of flux distributions taken by the metabolic network. Thus, after the introduction of the genes required for the biosynthesis of higher alcohols, productivity could be improved by gene deletions that restrict the range of flux distributions to produce the target alcohols (Figure 2). However, because the central metabolism in S. cerevisiae has limited flexibility, further gene deletions after the introduction of foreign genes 


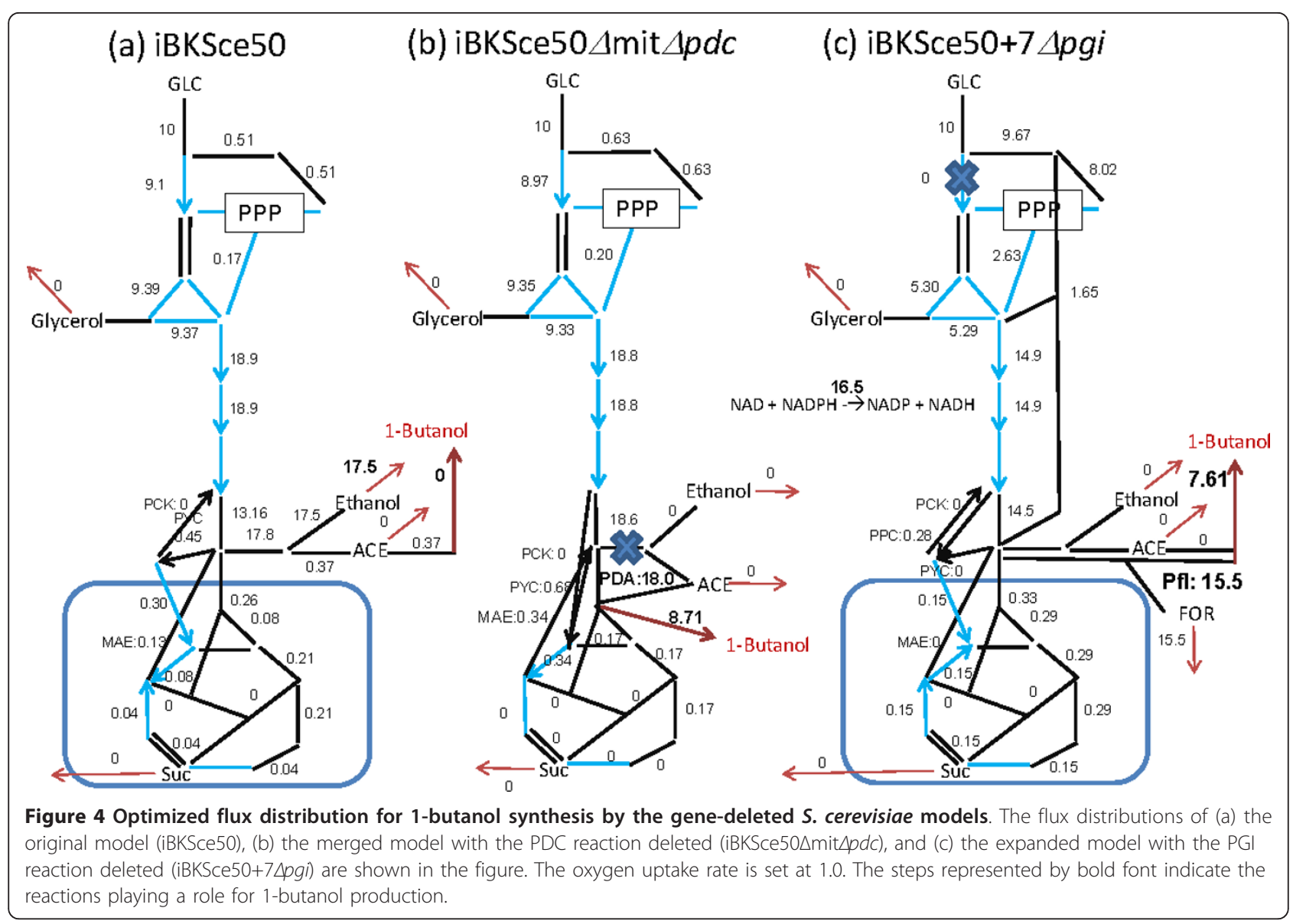

usually results in a serious reduction in cell growth (Figure 2 and 3 ). These results suggest that the strategy employed for the metabolic engineering of $E$. coli would be ineffective for S. cerevisiae.

The FBA-based metabolic simulations performed in this study, however, ignored the detailed aspects of metabolic regulations [30,47]. Because the kinetics of enzymatic reactions and the regulation of those activities were not considered, the results of the FBA-based analysis often over- or underestimated the role of metabolic pathways. This means that the FBA-based metabolic simulations could not predict the exact behavior of the metabolism of the microbe. To avoid these problems, metabolic performances derived from central metabolic networks were evaluated by employing the backbone metabolic models. Additionally, the production of higher alcohols in all single, double, triple, and quadruple deletion mutants were comprehensively determined to compare the overall trends of the metabolic performances of E. coli and S. cerevisiae. A possible drawback of the backbone model is the oversimplification of the metabolic network by omitting important metabolic functions. Although the details of yeast metabolism remain unclear, we reconstructed the backbone model by considering various studies and database informations [35-44].

\section{Conclusions}

If the stylized analysis performed in this study bears any resemblance to the real metabolism of E. coli and S. cerevisiae, the implications of this study for metabolic engineering of $S$. cerevisiae are radical. Due to its limited flexibility, the central metabolism of $S$. cerevisiae could not reach the flux distributions required for the effective production of higher alcohols. The comparison of the properties of metabolic network indicated that the cell compartmentalization and the lack of several shortcut reactions are possible causes of the low flexibility (Table 2 ). This implies that the functionality of the central metabolism of S. cerevisiae has to be supplemented by the addition of reactions (Table 2). Thus, the metabolic simulation demonstrated that the modified backbone model of $S$. cerevisiae possessing 7 E. coli reactions (iBKSce50+7) and the merged compartment (iBKSce $50 \Delta$ mit) had excellent production capabilities for the target alcohols (Figure 2b, d and 2f). Although the 
conclusion indicates a theoretical possibility for higher alcohol production, it is suggested that further modification of yeast metabolism requires the introduction of multiple genes and the coordinated regulation of their expression (Figure 4). Although the introduction of a single reaction to the central metabolism of $S$. cerevisiae has been attempted $[58,59]$, an innovative engineering methodology is required for implementing the strategy to the actual $S$. cerevisiae metabolism. This is the next challenge for the synthetic bioengineering of S. cerevisiae for the efficient production of higher alcohols.

\section{Methods}

\section{Model construction}

The genome-scale models of E. coli (iJR904 GSM/GPR) [45] and S. cerevisiae (iMM904)[46] were downloaded from http://gcrg.ucsd.edu. The backbone models of $E$. coli (iBKEco52) and S. cerevisiae (iBKSce50) were reconstructed using the information from the literature [35-41] and the databases including EcoCyC (http://ecocyc.org/)[42] and YEASTNET (http://www.comp-sysbio.org/yeastnet/)[43,44] (Supplementary Data S1). Elementary mode analysis was performed by the aid of METATOOL5.1 [60].

\section{FBA-based metabolic simulation}

The FBA-based metabolic simulation was performed via previously described methods [32] using MATLAB R2010b and glpk version 4.42 to perform the linear programming methods. The metabolic simulations were performed using the following procedure. (1) The linear programming was executed using the reactions for biomass production or growth rate as the objective function. The carbon source was fixed to be glucose consumed at $10 \mathrm{mmol} \mathrm{gDW}^{-1} \mathrm{~h}^{-1}$ in all analyses. (2) To avoid indefinite results, the flux for target production is minimized under the maximum growth rate condition. (3) The growth rates and target productivities were determined at 16 different oxygen uptake rates $(0.1,1,2 \ldots, 15 \mathrm{mmol})$. Those averages were considered the metabolic performance of the metabolic models. When the levels of growth speed were more than $1 \%$ of that of wild type, those deletion mutants were considered viable. The target-producing strains indicated the viable mutants producing target alcohols in yields exceeding $\mathrm{Y}_{\mathrm{Cmol} / \mathrm{Cmol} \text { glucose }}=0.01$. Because most deletions in the target-producing strains are silent or have adverse effects, we defined "proper" strains as target-producing strains in which all deletions contributed to the improvement of product yield. The network density and the characteristic path length were determined with the aid of Network Analyzer version 2.7 [57].

\section{Additional material}

Additional file 1: The E. coli backbone model iBKEco52 and the S. cerevisiae backbone model iBKSce 50 .

Additional file 2: Fermentation profiles of the in silico metabolic models.

Additional file 3: Pathways for higher alcohol biosyntheses inserted into the backbone models iBKEco52 and iBKSce50.

Additional file 4: The numbers of viable, target-producing, and proper deletion mutants obtained from all single, double, triple, and quadruple deletion mutants generated from the backbone models of $E$. coli (iBKEco52) and S. cerevisiae (iBKSce50,

iBKSce $50 \Delta$ mit, and iBKSce 50+7).

Additional file 5: Metabolic simulations of all single, double, triple, and quadruple deletion mutants generated from the backbone models of E. coli (iBKEco52) and S. cerevisiae (iBKSce50, iBKSce50 $\Delta$ mit, and iBKSce50+7).

Additional file 6: A comparison of the predicted growth rates of all single-reaction-deleted mutants with the experimental data.

\section{Acknowledgements}

This work was financed by Special Coordination Funds for Promoting Science and Technology, Creation of Innovation Centers for Advanced Interdisciplinary Research Areas (Innovative Bioproduction Kobe), MEXT, Japan.

\section{Author details}

${ }^{1}$ Organization of Advanced Science and Technology, Kobe University, Rokkodaicho, Nada-ku, Kobe, Hyogo, Japan. ²Department of Bioinformatic Engineering, Graduate School of Information Science and Technology, Osaka University, Yamadaoka, Suita, Osaka, Japan. ${ }^{3}$ Department of Chemical Science and Engineering, Graduate School of Engineering, Kobe University,

Rokkodaicho, Nada-ku, Kobe, Hyogo, Japan.

\section{Authors' contributions}

FM and CF performed the model construction and the metabolic simulations. FM, Jl, and TK analyzed the data. SH and AK designed the study. Jl, TK, and FM wrote the paper. All authors read and approved the final manuscript.

\section{Competing interests}

The authors declare that they have no competing interests.

Received: 25 June 2011 Accepted: 8 September 2011

Published: 8 September 2011

\section{References}

1. Connor MR, Atsumi S: Synthetic biology guides biofuel production. J Biomed Biotechnol 2010, pii: 541698.

2. Gheshlaghi R, Scharer JM, Moo-Young M, Chou CP: Metabolic pathways of clostridia for producing butanol. Biotechnol Adv 2009, 27:764-781.

3. Nielsen DR, Leonard E, Yoon SH, Tseng HC, Yuan C, Prather KL: Engineering alternative butanol production platforms in heterologous bacteria. Metab Eng 2009, 11:262-273.

4. Atsumi S, Cann AF, Connor MR, Shen CR, Smith KM, Brynildsen MP, Chou KJ, Hanai T, Liao JC: Metabolic engineering of Escherichia coli for 1butanol production. Metab Eng 2008, 10:305-311.

5. Clomburg JM, Gonzalez R: Biofuel production in Escherichia coli: the role of metabolic engineering and synthetic biology. Appl Microbiol Biotechnol 2010, 86:419-434.

6. Inui M, Suda M, Kimura S, Yasuda K, Suzuki H, Toda H, Yamamoto S, Okino S, Suzuki N, Yukawa H: Expression of Clostridium acetobutylicum butanol synthetic genes in Escherichia coli. Appl Microbiol Biotechnol 2008, 77:1305-1316.

7. Lee SY, Park JH, Jang SH, Nielsen LK, Kim J, Jung KS: Fermentative butanol production by Clostridia. Biotechnol Bioeng 2008, 101:209-228. 
8. Atsumi S, Hanai T, Liao JC: Non-fermentative pathways for synthesis of branched-chain higher alcohols as biofuels. Nature 2008, 451:86-89.

9. Shen CR, Lan El, Dekishima Y, Baez A, Cho KM, Liao JC: Driving forces enable high-titer anaerobic 1-butanol synthesis in Escherichia coli. Appl Environ Microbiol 2011, 77:2905-2915.

10. Shen C, Lan E, Dekishima Y, Baez A, Cho K, Liao J: High titer anaerobic 1butanol synthesis in Escherichia coli enabled by driving forces. Appl Environ Microbiol 2011, 77:2905-2915. 2011, 77:2905-2915.

11. Shen CR, Liao JC: Metabolic engineering of Escherichia coli for 1-butanol and 1-propanol production via the keto-acid pathways. Metab Eng 2008, 10:312-320.

12. Connor MR, Liao JC: Engineering of an Escherichia coli strain for the production of 3-methyl-1-butanol. Appl Environ Microbiol 2008, 74:5769-5775.

13. Atsumi S, WU TY, Machado IM, Huang WC, Chen PY, Pellegrini M, Liao JC: Evolution, genomic analysis, and reconstruction of isobutanol tolerance in Escherichia coli. Mol Sys Biol 2010, 6:449.

14. Cann AF, Liao JC: Pentanol isomer synthesis in engineered microorganisms. Appl Microbiol Biotechnol 2010, 85:893-899.

15. Cann AF, Liao JC: Production of 2-methyl-1-butanol in engineered Escherichia coli. Appl Microbiol Biotechnol 2008, 81:89-98.

16. Atsumi S, Liao JC: Directed evolution of Methanococcus jannaschii citramalate synthase for biosynthesis of 1-propanol and 1-butanol by Escherichia coli. Appl Environ Microbiol 2008, 74:7802-7808.

17. Atsumi S, Li Z, Liao JC: Acetolactate synthase from Bacillus subtilis serves as a 2-ketoisovalerate decarboxylase for isobutanol biosynthesis in Escherichia coli. Appl Environ Microbiol 2009, 75:6306-6311.

18. Atsumi S, Wu TY, Eckl EM, Hawkins SD, Buelter T, Liao JC: Engineering the isobutanol biosynthetic pathway in Escherichia coli by comparison of three aldehyde reductase/alcohol dehydrogenase genes. Appl Microbiol Biotechnol 2010, 85:651-657.

19. Connor MR, Cann AF, Liao JC: 3-Methyl-1-butanol production in Escherichia coli: random mutagenesis and two-phase fermentation. Appl Microbiol Biotechnol 2010, 86:1155-1164

20. Inokuma K, Liao JC, Okamoto M, Hanai T: Improvement of isopropanol production by metabolically engineered Escherichia coli using gas stripping. J Biosci Bioeng 2010, 110:696-701.

21. Huo YX, Cho KM, Rivera JG, Monte E, Shen CR, Yan Y, Liao JC: Conversion of proteins into biofuels by engineering nitrogen flux. Nature Biotechnol 2011, 29:346-351.

22. Baez A, Cho KM, Liao JC: High-flux isobutanol production using engineered Escherichia coli: a bioreactor study with in situ product removal. Appl Microbiol Biotechnol 2011, 90:1681-1690.

23. Jia $K$, Zhang $Y$, Li Y: Systematic engineering of microorganisms to improve alcohol tolerance. Eng Life Sci 2010, 10:422-429.

24. Hong ME, Lee KS, Yu BJ, Sung YJ, Park SM, Koo HM, Kweon DH, Park JC, Jin YS: Identification of gene targets eliciting improved alcohol tolerance in Saccharomyces cerevisiae through inverse metabolic engineering. J Biotechnol 2010, 149:52-59.

25. Fischer CR, Klein-Marcuschamer D, Stephanopoulos G: Selection and optimization of microbial hosts for biofuels production. Metab Eng 2008, 10:295-304.

26. Hirasawa T, Yoshikawa K, Nakakura Y, Nagahisa K, Furusawa C, Katakura Y, Shimizu H, Shioya S: Identification of target genes conferring ethanol stress tolerance to Saccharomyces cerevisiae based on DNA microarray data analysis. J Biotechnol 2007, 131:34-44.

27. Yoshikawa K, Tanaka T, Furusawa C, Nagahisa K, Hirasawa T, Shimizu H: Comprehensive phenotypic analysis for identification of genes affecting growth under ethanol stress in Saccharomyces cerevisiae. FEMS Yeast Res 2009, 9:32-44.

28. Matsui K, Teranishi S, Kamon S, Kuroda K, Ueda M: Discovery of a modified transcription factor endowing yeasts with organic-solvent tolerance and reconstruction of an organic-solvent-tolerant Saccharomyces cerevisiae strain. Appl Environ Microbiol 2008, 74:4222-4225.

29. Steen EJ, Chan R, Prasad N, Myers S, Petzold CJ, Redding A, Ouellet M, Keasling JD: Metabolic engineering of Saccharomyces cerevisiae for the production of n-butanol. Microb Cell Fact 2008, 7:36

30. Orth JD, Thiele I, Palsson BO: What is flux balance analysis? Nature Biotech 2010, 28:245-248.

31. Lee JM, Gianchandani EP, Papin JA: Flux balance analysis in the era of metabolomics. Brief Bioinform 2006, 7:140-150.
32. Shinfuku Y, Sorpitiporn N, Sono M, Furusawa C, Hirasawa T, Shimizu H: Development and experimental verification of a genome-scale metabolic model for Corynebacterium glutamicum. Microb Cell Fact 2009, 8:43.

33. Trinh $C T$, Wlaschin A, Srienc F: Elementary mode analysis: a useful metabolic pathway analysis tool for characterizing cellular metabolism. Appl Microbiol Biotechnol 2009, 81:813-826.

34. Park JM, Kim TY, Lee SY: Constraints-based genome-scale metabolic simulation for systems metabolic engineering. Biotechnol Adv 2009, 27:979-988.

35. Trinh CT, Unrean P, Srienc F: Minimal Escherichia coli cell for the most efficient production of ethanol from hexoses and pentoses. Appl Environ Microbiol 2008, 74:3634-3643.

36. Nookaew I, Meechai A, Thammarongtham C, Laoteng K, Ruanglek V, Cheevadhanarak S, Nielsen J, Bhumiratana S: Identification of flux regulation coefficients from elementary flux modes: A systems biology tool for analysis of metabolic networks. Biotechnol Bioeng 2007, 97:1535-1549.

37. Pitkanen JP, Aristidou A, Salusjarvi L, Ruohonen L, Penttila M: Metabolic flux analysis of xylose metabolism in recombinant Saccharomyces cerevisiae using continuous culture. Metab Eng 2003, 5:16-31.

38. Wahlbom CF, Eliasson A, Hahn-Hagerdal B: Intracellular fluxes in a recombinant xylose-utilizing Saccharomyces cerevisiae cultivated anaerobically at different dilution rates and feed concentrations. Biotechnol Bioeng 2001, 72:289-296.

39. Nissen TL, Schulze U, Nielsen J, Villadsen J: Flux distributions in anaerobic, glucose-limited continuous cultures of Saccharomyces cerevisiae. Microbiology 1997, 143(Pt 1):203-218.

40. Jeppsson M, Johansson B, Hahn-Hagerdal B, Gorwa-Grauslund MF: Reduced oxidative pentose phosphate pathway flux in recombinant xyloseutilizing Saccharomyces cerevisiae strains improves the ethanol yield from xylose. Appl Environ Microbiol 2002, 68:1604-1609.

41. Gombert AK, Moreira dos Santos M, Christensen B, Nielsen J: Network identification and flux quantification in the central metabolism of Saccharomyces cerevisiae under different conditions of glucose repression. J Bacteriol 2001, 183:1441-1451.

42. Keseler IM, Collado-Vides J, Santos-Zavaleta A, Peralta-Gil M, Gama-Castro S, Muniz-Rascado L, Bonavides-Martinez C, Paley S, Krummenacker M, Altman T, et al: EcoCyc: a comprehensive database of Escherichia coli biology. Nucleic Acids Res 2010, 39:D583-590.

43. Dobson PD, Smallbone K, Jameson D, Simeonidis E, Lanthaler K, Pir P, Lu C, Swainston N, Dunn WB, Fisher $P$, et al: Further developments towards a genome-scale metabolic model of yeast. BMC Syst Biol 2010, 4:145

44. Herrgard MJ, Swainston N, Dobson P, Dunn WB, Arga KY, Arvas M, Bluthgen N, Borger S, Costenoble R, Heinemann M, et al: A consensus yeast metabolic network reconstruction obtained from a community approach to systems biology. Nature Biotech 2008, 26:1155-1160.

45. Reed JL, Vo TD, Schilling CH, Palsson BO: An expanded genome-scale model of Escherichia coli K-12 (iJR904 GSM/GPR). Genome Biol 2003, 4 R54.

46. Mo ML, Palsson BO, Herrgard MJ: Connecting extracellular metabolomic measurements to intracellular flux states in yeast. BMC Syst Biol 2009, 3:37.

47. Feist AM, Palsson BO: The biomass objective function. Curr Opin Microbiol 2010, 13:344-349

48. Fong SS, Burgard AP, Herring CD, Knight EM, Blattner FR, Maranas CD, Palsson BO: In silico design and adaptive evolution of Escherichia coli for production of lactic acid. Biotechnology Bioeng 2005, 91:643-648.

49. Kennedy CJ, Boyle PM, Waks Z, Silver PA: Systems-level engineering of nonfermentative metabolism in yeast. Genetics 2009, 183:385-397.

50. Alper H, Miyaoku K, Stephanopoulos G: Construction of lycopeneoverproducing $\mathrm{E}$. coli strains by combining systematic and combinatorial gene knockout targets. Nature Biotech 2005, 23:612-616.

51. Burgard AP, Pharkya P, Maranas CD: Optknock: a bilevel programming framework for identifying gene knockout strategies for microbial strain optimization. Biotechnology Bioeng 2003, 84:647-657.

52. Hua Q, Joyce AR, Fong SS, Palsson BO: Metabolic analysis of adaptive evolution for in silico-designed lactate-producing strains. Biotechnology Bioeng 2006, 95:992-1002

53. Engel SR, Balakrishnan R, Binkley G, Christie KR, Costanzo MC, Dwight SS, Fisk DG, Hirschman JE, Hitz BC, Hong EL, et al: Saccharomyces Genome 
Database provides mutant phenotype data. Nucleic Acids Res 2010, 38 : D433-436.

54. Warringer J, Ericson E, Fernandez L, Nerman O, Blomberg A: Highresolution yeast phenomics resolves different physiological features in the saline response. Proc Natl Acad Sci U S A 2003, 100:15724-15729.

55. Breslow DK, Cameron DM, Collins SR, Schuldiner M, Stewart-Ornstein J, Newman HW, Braun S, Madhani HD, Krogan NJ, Weissman JS: A comprehensive strategy enabling high-resolution functional analysis of the yeast genome. Nature Methods 2008, 5:711-718.

56. Stelling J, Klamt S, Bettenbrock K, Schuster S, Gilles ED: Metabolic network structure determines key aspects of functionality and regulation. Nature 2002, 420:190-193.

57. Assenov Y, Ramirez F, Schelhorn SE, Lengauer T, Albrecht M: Computing topological parameters of biological networks. Bioinformatics 2008, 24:282-284.

58. Moreira dos Santos M, Raghevendran V, Kotter P, Olsson L, Nielsen J: Manipulation of malic enzyme in Saccharomyces cerevisiae for increasing NADPH production capacity aerobically in different cellular compartments. Metab Eng 2004, 6:352-363.

59. Waks Z, Silver PA: Engineering a synthetic dual-organism system for hydrogen production. Appl Environ Microbiol 2009, 75:1867-1875.

60. von Kamp A, Schuster S: Metatool 5.0: fast and flexible elementary modes analysis. Bioinformatics 2006, 22:1930-1931.

doi:10.1186/1475-2859-10-70

Cite this article as: Matsuda et al.: Engineering strategy of yeast metabolism for higher alcohol production. Microbial Cell Factories 2011 $10: 70$.

\section{Submit your next manuscript to BioMed Central} and take full advantage of:

- Convenient online submission

- Thorough peer review

- No space constraints or color figure charges

- Immediate publication on acceptance

- Inclusion in PubMed, CAS, Scopus and Google Scholar

- Research which is freely available for redistribution

Submit your manuscript at www.biomedcentral.com/submit 